To cite this article: Tikici D, Er S, Tez M. Comparison of American Society of Anesthesiology (ASA) and Charlson's Comorbidity Index (CCI) for Predicting Mortality in Emergency Colorectal Surgery. Turk J Clin Lab 2018; 9(3): 162-165.

-Orijinal Makale

\title{
Acil kolorektal cerrahi yapılan hastalarda mortaliteyi öngörmede Amerikan Anesteziyoloji Derneği Sınıflaması ve Charlson Komorbidite İndeksi'nin karşılaştırılması
}

\section{Comparison of American Society of Anesthesiology Classification and Charlson's Comorbidity Index for predicting mortality in emergency colorectal surgery}

Deniz TikiCi, Sadettin ER*, Mesut TEZ

Ankara Numune Training and Research Hospital, General Surgery, Department of Colorectal Surgery, Ankara/Turkey.

\section{öz}

Amaç: Acil kolorektal cerrahi yapılan hastalarda kolay uygulanabilir ve pratik olan ASA (Amerikan Anesteziyoloji Derneği Sınıflaması) ve CCI (Charlson Komorbidite İndeksi) gibi skorlama sistemlerini kullanarak preoperatif değerlendirme ile mortaliteyi öngörebilmeyi amaçladık.

Gereç ve Yöntemler: Ocak 2014-Aralık 2017 tarihleri arasında Sağlık Bilimleri Üniversitesi Ankara Numune Eğitim ve Araştırma Hastanesi Genel Cerrahi/Acil Cerrahi Kliniğine başvuran Acil Kolorektal Cerrahi (AKC) yapılan 198 hastanın verileri retrospektif olarak incelendi. Hastaların demografik özellikleri, klinik tanıları, uygulanan cerrahi tipi, postoperatif komplikasyonlar, komorbiditeler, ASA ve CCI skorları elektronik veri tabanından elde edildi.

Bulgular: 198 hastanın 95'i (\%47,9) kadın ve 103'ü $(\% 52,1)$ erkek idi. Ortanca yaş 62 (23 -93) olarak bulundu. 49(\% 24) hastada mortalite gelişti. Mortalite gelişen hastaların ortanca ASA skoru: 4, CCI: 5 ve mortalite gelişmeyen hastaların ortanca ASA skoru: 3, CCl: 5 idi. Mortalite için ASA skoru istatistiksel olarak anlamlı bulundu (ASA için $p=0,001$ ve CCl için $p=0,611$ ).

Sonuç: Bu çalışmanın, yüksek riskli hastaların yönetiminde pratik olarak kullanılabilecek basit ve yararlı veri sağladığına inanmaktayız. Acil kolorektal cerrahide, mortaliteyi öngörmede ASA skoru hızlı uygulanabilir ve yeterli bir skorlamadır.

Anahtar kelimeler: Mortalite, acil kolorektal cerrahi, ASA skoru ve CCI.

Sorumlu Yazar": Sadettin Er, Ankara Numune Training and Research Hospital, General Surgery, Department of Colorectal Surgery, Ankara/Turkey. 


\title{
ABSTRACT
}

\begin{abstract}
Aim: We aimed to predict mortality by using preoperative evaluation scoring systems such as ASA (American Society of Anesthesiologists Classification) and CCI (Charlson Comorbidity Index), which are easily practicable in emergency colorectal surgery patients.
\end{abstract}

Material and Methods: The data of 198 patients with Emergency Colorectal Surgery (ECS) who applied to Ankara Numune Training and Research Hospital General Surgery / Emergency Surgery Clinic of Health Sciences University between January 2014 and December 2017 were retrospectively analyzed. Demographic characteristics, clinical diagnoses, type of surgery, postoperative complications, comorbidities, ASA and CCI scores were obtained from electronic databases.

Results: Of the 198 patients, 95 (47.9\%) were female and 103 (52.1\%) were male. The median age was 62 (23-93). 49 (\%24) mortality was seen in patients. The median ASA score was 4, the CCI was 5 in patients with mortality; and the median ASA score was $3, \mathrm{CCl}$ was 5 in patients with no mortality. The ASA score for predicting mortality was found statistically significant (respectively $\mathrm{p}$ value for ASA and $\mathrm{CCl} ; 0.001$ and 0.611 ).

Conclusion: We believe this study provides simple and useful data that can be used practically in the management of highrisk patients. In emergency colorectal surgery, ASA score is quickly applicable and adequate score for predicting mortality.

Keywords: Mortality, emergenct colorectal surgery, ASA score and CCI.

\section{Giriş}

Acil kolorektal cerrahi (AKC) yapılan hastalarda, cerrahi tekniklerdeki gelişmelere rağmen morbidite ve mortalite oranları halen yüksek seyretmektedir. AKC nedenleri arasında en sık kolorektal kanserler görülmektedir. Bunun yanı sıra volvulus, mezenter iskemi, divertiküler hastalık, travmatik ve non-travmatik perforasyonlar AKC nedenleri arasındadır. Kolorektal obstrüktif kitleler acil servise en sık başvuru nedenleri olarak görülmektedir [1]. Literatüre bakıldığında AKC yapılan hastalarda mortalite oranları \% 2.3-\%80 arasında değişmektedir $[2,3]$. Bu geniş mortalite aralığl; acil cerrahi nedeni, komorbidite ve cerrahinin yapıldığı kliniğin deneyimine göre değişmektedir. Cerrahi veya medikal tedavi alan hastalarda, preoperatif değerlendirme ve yoğun bakım takibinde kullanılmak üzere, morbidite ve mortaliteyi öngörebilmek için birçok skorlama sistemleri geliştirilmiştir. POSSUM (Physiological and Operative Severity Score for the enUmeration of Mortality and Morbidity), P-POSSUM (Portsmouth-Physiological and Operative Severity Score for the enUmeration of Mortality and Morbidity), CRPOSSUM (Colorectal-Physiological and Operative Severity Score for the enUmeration of Mortality and Morbidity), SOFA (Sepsis related Organ Failure Assessment), APACHE II (Acute Physiology and Chronic Health Evaluation), ASA (American Society of Anesthesiologists Classification) ve CCI (Charlson's Comorbidity Index) gibi skorlama sistemleri geliştirilmiştir. Literatürde bu skorlama sistemlerinin etkinliklerini değerlendiren ve birbirlerine üstünlüklerini kıyaslayan birçok çalışma mevcuttur. Mortalite ve morbiditeyi öngörüde, CCl yaygın olarak
kullanılmaktadır[4].Kolayvehızlıuygulanabilirolması ve mortaliteyi öngörmedeki başarısı yaygın olarak kullanılmasını sağlamıştır.

ASA sınıflaması daha çok anestezistler tarafından preoperatif değerlendirmede kullanılmaktadır. ASA sınıflaması yapılırken komorbiditenin ciddiyet düzeyine bakılmaktadır. ASA'nın kolay uygulanabilir olması önemli bir avantajıdır. ASA sınıflamasında, CCl'den farklı olarak komorbiditenin ciddiyeti ile skorlama arasında bir ilişki mevcuttur. Acil kolorektal cerrahi yapılan hastalarda kolay uygulanabilir ve pratik olan ASA ve CCl gibi skorlama sistemlerini kullanarak preoperatif değerlendirme ile mortaliteyi öngörebilmeyi amaçladık.

Tercih edilecek skorlama sistemi ile mortaliteyi öngörmenin amacl; uygulanacak cerrahi tipine karar vermede yardımcı olması, operasyon süresi ile ilişkili olabilecek morbidite ve mortaliteyi azaltması ve postoperatif hasta takibinde yol gösterici olmasıdır.

\section{Gereç ve Yöntem}

\section{Hasta Verileri}

Ocak 2014 ile Aralık 2017 tarihleri arasında Sağlık Bilimleri Üniversitesi Ankara Numune Eğitim ve Araştırma Hastanesi Genel Cerrahi/Acil Cerrahi Kliniğine başvuran AKC yapılan 198 hastanın verileri retrospektif olarak incelendi. Hastaların demografik özellikleri, klinik tanıları, uygulanan cerrahi tipi, postoperatif komplikasyonlar, komorbiditeler, ASA ve CCI skorları elektronik veri tabanından elde edilerek bulundu.

\section{İstatiksel analiz}

İstatistiksel analizler SPSS, Windows 12.0 sürümü kullanılarak yapıldı. Non-parametrik sayısal veriler medyan (Interquartile 
range $=$ IQR) olarak verildi. Kategorik değişkenler yüzde (\%) olarak ifade edildi. Sayısal değişkenler Mann-Whitney U testi ve kategorik değişkenler Ki-kare veya Fisher exact testi ile karşılaştırıldı. $\mathrm{P}<0,05$ istatiksel olarak anlamlı kabul edildi.

\section{Bulgular}

Çalışmaya dahil edilen 198 hastanın 95'i $(\% 47,9)$ kadın ve 103 'ü $(\% 52,1)$ erkek idi. Ortanca yaş 62 (23-93) olarak bulundu. $49(\% 24)$ hastada mortalite gelişti. Mortalite gelişen hastaların ortanca ASA skoru: 4, CCl: 5 ve mortalite gelişmeyen hastaların ortanca ASA skoru: 3, CCl: 5 idi. Mortalite için ASA skoru istatistiksel olarak anlamlı bulundu( $A S A$ için $P=0,001$ ve $C C l$ için $\mathrm{P}=0,611)$. (Tablo-1). Hastalara ait cerrahi yöntem, tanı ve ostomi verileri Tablo-2'de özetlenmiştir.

\begin{tabular}{|c|c|c|c|c|c|}
\hline \multicolumn{6}{|c|}{$\begin{array}{l}\text { Tablo -1: Mortalite ve mortalite gelişmeyen hastaların ASA } \\
\text { ve CCI deăerleri }\end{array}$} \\
\hline \multirow{5}{*}{$\begin{array}{l}\text { ASA } \\
\text { Skoru }\end{array}$} & & $\begin{array}{l}\text { Non-Mor- } \\
\text { talite }(n=149)\end{array}$ & $\begin{array}{l}\text { Mortalite } \\
(\mathrm{n}=49)\end{array}$ & $\begin{array}{c}\text { Total } \\
(\mathrm{n}=198)\end{array}$ & $\begin{array}{l}P \\
\text { değeri }\end{array}$ \\
\hline & ASA I & $4(\% 100)$ & $0(\% 0)$ & $4(\% 2)$ & \multirow{5}{*}{, $001^{*}$} \\
\hline & ASA II & $55(\% 93.3)$ & $4(\% 6.7)$ & $59(\% 29.8)$ & \\
\hline & \multirow{2}{*}{ ASA III } & 69 (\%84.2) & $13(\% 15.8)$ & $82(\% 41.4)$ & \\
\hline & & $20(\% 47.6)$ & $22(\% 52.4)$ & $42(\% 21.2)$ & \\
\hline & ASA V & $1(\% 9)$ & $10(\% 91)$ & $11(\% 0.5)$ & \\
\hline $\mathrm{CCl}$ & $\mathrm{CCl}<6$ & $84(\% 76.4)$ & 26 (\%23.6) & $110(\% 55.5)$ & \\
\hline Skoru & $\mathrm{CCl} \geq 6$ & 65 (\%73.9) & $23(\% 26.1)$ & $88(\% 45.5)$ & NS \\
\hline $\begin{array}{l}\text { NS: is } \\
*: \text { Ista }\end{array}$ & $\begin{array}{l}\text { nericanS } \\
\text { arlsonCo } \\
\text { iksel olar }\end{array}$ & $\begin{array}{l}\text { xiety of Anes } \\
\text { norbidity Ind } \\
\text { ak anlamlı dec } \\
\text { anlamlı }\end{array}$ & thesiol & & \\
\hline
\end{tabular}

\begin{tabular}{|c|c|c|}
\hline $\begin{array}{l}\text { Tablo-2: Hastala } \\
\text { veriler }(n=198)\end{array}$ & arın cerrahi yöntem, tanı ve osto & milerine ait \\
\hline & Sağ hemikolektomi & $87(\% 43,9)$ \\
\hline & Sol hemikolektomi & $32(\% 16,2)$ \\
\hline & Sigmoid kolektomi & $21(\% 10,6)$ \\
\hline & Transvers kolektomi & $2(\% 1)$ \\
\hline & Low anterior rezeksiyon & $21(\% 10,6)$ \\
\hline Cerrani rontem & Hartmann kolostomi & $1(\% 0,5)$ \\
\hline & Total kolektomi & $5(\% 2,5)$ \\
\hline & Subtotal kolektomi & $7(\% 3,5)$ \\
\hline & Anterior rezeksiyon & $22(\% 11,1)$ \\
\hline & Kolorektal kanserler & $110(\% 55,6)$ \\
\hline & Divertikülit & $9(\% 4,5)$ \\
\hline & Mezenter iskemi & $34(\% 17,2)$ \\
\hline & Volvulus & $13(\% 6,6)$ \\
\hline Ianı & Travmatik kolon perforasyonu & $7(\% 3,5)$ \\
\hline & Non-travmatik kolon perforasyonu & $12(\% 6,1)$ \\
\hline & Strangüle herni & $2(\% 1)$ \\
\hline & Diğerleri & $11(\% 5,6)$ \\
\hline & Var & $123(\% 62,1)$ \\
\hline & Yok & $75(\% 37,9)$ \\
\hline
\end{tabular}

\section{Tartışma}

Günümüz klinik uygulama ve araştırmalarını etkileyen komorbidite, sağlıksistemleri reformu vefonlama modellerinde önemli bir durumdur [5]. Bununla birlikte, komorbiditeyi değerlendirmek için en etkili yöntem konusunda halen bir fikir birliği yoktur [6]. CCl, düşük maliyet, kolay uygulanabilirlik ve hızlı yorumlama gibi özelliklere sahiptir [7]. Aynı şekilde, preoperatif risk değerlendirmesinde ASA sınıflaması da kolay uygulanabilir olması nedeniyle yaygın olarak kullanılmaktadır.

Yüksek riskli hastaların postoperatif morbidite ve mortalitelerinin saptanması zordur. Klinik pratikte preoperatif motalitenin öngörülmesi hasta yönetimi açısından son derece önemlidir. Bunun için çeşitli skorlama sistemleri geliştirilmiştir. ASA ve $\mathrm{CCl}$ de bu skorlama sistemlerinden birkaçını oluşturur. AKC hastaları genellikle ileri yaş ve birçok komorbiditeye sahip hasta grubunu oluşturmaktadır. Mortalite için tanımlanabilecek ve modifiye edilebilecek perioperatif risk faktörlerinden birisi acil cerrahi girişimlerdir. Bu girişimlerde hastanın ve ekibin hazırlanma süresi oldukça kısa olduğundan mortalite oranları çok yüksektir [8]. Buna göre preoperatif mortalite tahmini hasta açısından yoğun bakım ihtiyacı, radikal cerrahi gerekip/gerekmediği ve minimal cerrahi yaklaşımlar konusunda cerraha rehberlik edebilir.

ASA fiziksel durum sınıflandırması, cerrahi hastalar için preoperatif değerlendirmenin en yaygın kullanılan standart skorlarından birisidir. İyi bir klinik değerlendirme sağlayan ASA sınıflandırmasına göre, dereceleri 4 ve 5 olan hastalarda mortalitenin çok yüksek olduğu bilinmektedir [9]. Leonardo C. Duraes ve ark.'ın [10] yapmış olduğu çalışmada 1 yıllık mortalitede ASA bağımsız değişken olarak bulunmuştur. Özellikle acil olgularda hastanın genel kondisyonu kötü ise ve komorbidite mevcudiyeti durumunda ASA skoru artmaktadır ve buna bağlı olarak morbidite ve mortalite oranlarında artış görülmektedir.

Bu skorlama sistemlerinden $\mathrm{CCl}$, birçok farklı klinik durumda sonuçları değerlendirmek için kullanılan fiziksel sağlık ölçümlerinden biridir. Ciddi organ disfonksiyonu varlığı; tedavi planlamasını ve tedavinin sonucunu etkilemektedir [11]. Çeşitli benign ve malign hastalıklarla ilgili daha önce yapılan çalışmalarda daha yüksek komorbidite yükü olan hastalarda daha kısa süreli sağkalım olduğu gösterilmiştir [12-14].

Mortalitenin CCI gibi diğer, belki de daha sofistike araçların yerine ASA sınıflandırmasına dayandığı gösterilmiştir [10]. Çalışmalarda; acil cerrahi yapılan ve daha yüksek ASA skorlu hastalar, yüksek postoperatif mortalite ile ilişkilendirilmiştir [15]. Başka bir çalışmada; ASA $\geq 11$ olması, postoperatif mortalite için bağımsız risk faktörü olduğu gösterilmiştir [16]. Yaptığımız 
çalışmada, ASA skoru CCI'ya göre mortaliteyi öngörmede istatiksel olarak anlamlı bulunmuştur $(p=0,001)$.

Sonuç olarak; bu çalışmanın, yüksek riskli hastaların yönetiminde pratik olarak kullanılabilecek basit ve yararlı veri sağladığına inanmaktayız. Acil kolorektal cerrahide, mortaliteyi öngörmede ASA skoru hızlı uygulanabilir ve yeterli bir skorlamadır.

\section{Çıkar çatışması / finansal destek beyanı}

Bu yazıdaki hiçbir yazarın herhangi bir çıkar çatışması yoktur. Yazının herhangi bir finansal desteği yoktur

\section{Kaynaklar}

1. Jiang J, Lan Y, Lin T, Chen $\mathrm{W}$ et al. Primary vs. delayed resection for obstructive left-sided colorectal cancer: impact of surgery on patient outcome. Diseases of the Colon \& Rectum 2008; 51: 306-11.

2. Fazio VW, Tekkis PP, Remzi F, Lavery IC. Assessment of operative risk in colorectal cancer surgery: the Cleveland Clinic Foundation colorectal cancer model. Diseases of the Colon \& Rectum 2004; 47: 2015-24

3. Jrvinen O, Laurikka J, Salenius J, Tarkka M, editors. Acute intestinal ischaemia. A review of 214 cases. Annales chirurgiae et gynaecologiae; 1994.

4. Charlson ME, Pompei P, Ales KL, MacKenzie CR. A new method of classifying prognostic comorbidity in longitudinal studies: development and validation. Journal of chronic diseases 1987 40: 373-83.

5. Roffman CE, Buchanan J, Allison GT. Predictors of non-use of prostheses by people with lower limb amputation after discharge from rehabilitation: development and validation of clinical prediction rules. Journal of physiotherapy 2014; 60: 224-31.

6. Molto A, Dougados M. Comorbidity indices. Clin Exp Rheumatol 2014; 32: 131-34.
7. Hall WH, Ramachandran R, Narayan S, Jani AB, Vijayakumar S. An electronic application for rapidly calculating Charlson comorbidity score. BMC cancer 2004; 4: 94.

8. Erden A, Uzun Ş, Turgut $H$, Aypar Ü. Yaşlı hastalarda anestezi. Akademik Geriatri Dergisi 2009; 1: 162-66.

9. Kettunen J, Paajanen $\mathrm{H}$, Kostiainen S. Emergency abdominal surgery in the elderly. Hepato-gastroenterology. 1995; 42: 106-8.

10. Duraes LC, Stocchi L, Dietz D et al. The disproportionate effect of perioperative complications on mortality within 1 year after colorectal cancer resection in octogenarians. Annals of surgical oncology 2016; 23: 4293-301.

11. Chang C-M, Yin W-Y, Wei C-K et al. Adjusted age-adjusted Charlson Comorbidity Index score as a risk measure of perioperative mortality before cancer surgery. PLoS One 2016; 11: 0148076.

12. Mayr R, May M, Martini T et al. Comorbidity and performance indices as predictors of cancer-independent mortality but not of cancer-specific mortality after radical cystectomy for urothelial carcinoma of the bladder. European urology 2012; 62: 662-70.

13. Ng ACC, Chow V, Yong ASC, Chung T, Kritharides L. Prognostic impact of the Charlson comorbidity index on mortality following acute pulmonary embolism. Respiration 2013; 85: 408-16.

14. Schneider EB, Hyder O, Brooke BS et al. Patient readmission and mortality after colorectal surgery for colon cancer: impact of length of stay relative to other clinical factors. Journal of the American College of Surgeons. 2012; 214: 390-98.

15. Smith J, Tilney $\mathrm{H}$, Heriot A et al. Social deprivation and outcomes in colorectal cancer. British Journal of Surgery: Incorporating European Journal of Surgery and Swiss Surgery. 2006; 93: 1123-31.

16. Iversen LH. Aspects of survival from colorectal cancer in Denmark. Danish medical journal. 2012; 59: 4428 